Stabile Angina pectoris

\section{Sport statt Stent}

Eine durch körperliche Bewegung geförderte endotheliale NO-Freisetzung entfaltet bei KHK-Patienten ihre nützlichen Effekte nicht allein durch eine Gefäßweitstellung, sondern fördert zusätzlich Ausbildung und Wachstum kardialer Kollateralgefäße, ist sich Priv.-Doz. Dr. Ivo Buschmann von der Berliner Charité sicher.

Das Gefäßendothel ist maßgeblich für die Anpassung des regionalen Blutflusses an den Sauerstoffbedarf zuständig. Ist dieser Mechanismus gestört, droht etwa auch eine kardiale Sauerstoffunterversorgung - sprich eine Angina pectoris.

Nicht in jedem Fall muss man einem solchen Geschehen durch den Einbau eines Korornarstents begegnen, hat Priv.-Doz. Dr. Stephan Gielen vom Universitätsklinikum Halle deutlich gemacht. In vielen Konstellationen erweist sich ein maßvolles körperliches Training in Kombination mit einer optimalen medikamentösen Therapie bei KHK-Patienten als mindestens so effektiv wie eine interventionelle Therapie.

Zumindest im Rahmen der ärztlich überwachten kardialen Rehabilitation lässt sich der sportlichen Leistungsfähigkeit der Patienten auch durch eine (vorübergehende) unterstützende Gabe von Nitrogylcerinpräparaten wie Glyceroltrinitrat (Nitrolingual akut ${ }^{\circledR}$ Spray) auf die Sprünge helfen. Über eine NO-vermittelte Vasodilatation zielt diese Maßnahme auf eine Senkung von Vor- und Nachlast und damit auf die Verhinderung einer belastungsinduzierten Angina pectoris. Dass durch einen entsprechenden Gebrauch die Belastungsdauer erhöht und die Dauer bis zum Erreichen der Anginaschwelle verlängert werden kann, konnten Untersuchungen zeigen. (urm) I

II Satellitensymposium bei der 15. Frühjahrstagung des Herzzentrums Leipzig, 11. Mai 2012 (Veranstalter: Pohl Boskamp)

\title{
Nierenfunktionsstörung
}

\section{Niedrige LDL-Zielwerte anstreben}

Nach den neuen ESC/EAS-Leitlinien zum Dyslipidämie-Management werden nun auch Patienten mit moderater bis schwerer Einschränkung der Nierenfunktion der Hochrisikogruppe zugeordnet. Dies bedeutet: Das LDLCholesterin sollte möglichst unter 70 $\mathrm{mg} / \mathrm{dl}$ gedrückt werden.

Dass eine chronische Niereninsuffizienz die kardiovaskuläre Ereignisrate erhöht, ist keine neue Erkenntnis. So fanden etwa Go und Mitarbeiter schon vor geraumer Zeit einen Zusammenhang zwischen nachlassender Nierenfunktion und kardiovaskulärer Ereignisrate, Gesamtmortalität und Hospitalisierungsrate. Genauso wie eine KHK oder ein Diabetes mellitus mit Endorganschäden qualifiziert auch eine eingeschränkte Nierenfunktion zur Aufnahme in die Hochrisikogruppe.
Bei dem Bemühen, das LDL-Cholesterin bei nierenschwachen Patienten auf den angepeilten Wert $<70 \mathrm{mg} / \mathrm{dl}$ zu bringen, erweist sich die kombinierte Gabe von $10 \mathrm{mg}$ Ezetimib plus $20 \mathrm{mg}$ Simvastatin als aussichtsreiche Strategie. So jedenfalls zeigen es die von Prof. Christian Schneider von der Kölner PAN-Klinik Neumarkt vorgestellten Ergebnisse der SHARP-Studie, in die 9270 Patienten mit eingeschränkter Nierenfunktion, davon 3023 dialysepflichtig, aufgenommen wurden. Unter der Kombination von Ezetimib und Simvastatin (Inegy ${ }^{\circledR}$ ) konnte das LDL-Cholesterin von initial $108 \mathrm{mg} / \mathrm{dl}$ auf $76 \mathrm{mg} / \mathrm{dl}$ gesenkt werden. Gegenüber Placebo ergab sich eine LDL-Reduktion um $29,6 \%$.

(urm) II

II Kardiologen-Akademie 2012, Berlin, 4./5. Mai 2012 (Veranstalter: MSD in Kooperation mit dem BNK)

\section{Hypertonie}

\section{Mehr Erfolg durch einfache Therapie}

\section{Diabetes mellitus erhöht das Risiko für kardiovaskuläre Ereignisse bei Hyper- tonikern erheblich. Dieses Risiko wird allerdings in der Therapie oft vernach- lässigt. Signifikant gesenkt werden kann es durch eine leitliniengerechte Antihypertensiva-Kombination wie Perindopril/Indapamid weitaus effek- tiver als etwa durch normnahe Einstel- lung des Blutzuckers. Der Blutdruck sollte aber Werte von $120 / 70 \mathrm{mmHg}$ nicht unterschreiten.}

Unter dem Eindruck neuer Studienergebnisse wie INVEST und ACCORD haben sich inzwischen die Zielwerte der Blutdrucksenkung für KHK-Patienten und Diabetiker verändert. Denn es hat sich gezeigt, dass bei Unterschreiten von 120/70 mmHg das Risiko für kardiovaskuläre Ereignisse wieder steigt. Die Leitlinien empfehlen deshalb, dass diese Patientengruppen einen Blutdruck von $<130 / 80 \mathrm{mmHg}$ erreichen sollten, sagte Prof. Rainer Kolloch, Bielefeld. Dieses Ziel ist in der
Praxis schon schwer genug realisierbar, ebenso wie der Anspruch, alle Hypertoniker unter $140 / 90 \mathrm{mmHg}$ zu bringen, so Kolloch. Ein einfacheres Therapieregime, insbesondere die Nutzung fixer Kombinationen, kann dazu beitragen, die Erfolgsquote zu steigern.

Seit der HYVET-Studie wissen wir auch, dass hochbetagte Patienten (> 80 Jahre) von einer Blutdrucksenkung profitieren. Das Risiko für tödliche und nicht tödliche Schlaganfälle nahm in dieser Studie unter Perindopril/Indapamid (BiPreterax ${ }^{\oplus} \mathrm{N}$ ) signifikant ab. Nach den Leitlinien der deutschen Hochdruckliga sollte der systolische Blutdruck von sehr alten Patienten aufWerte unter 150 $\mathrm{mmHg}$ gesenkt werden, erklärte Dr. Ulrich Kintscher, Berlin.

(abi)

II Symposium des Arbeitskreises Hypertonie im BDI e.V., 118. Kongress der Deutschen Gesellschaft für Innere Medizin, Wiesbaden, 14.-17. April 2012 (mit Unterstützung von Servier) 